\title{
Numerical simulation analysis of rotary cup centrifugal blast furnace slag
}

\author{
Mengyuan Yang ${ }^{1,2}$, and Jingfu Wang ${ }^{1,2, *}$ \\ ${ }^{1}$ MOE Key Laboratory of Enhanced Heat Transfer and Energy Conservation, Beijing University of \\ Technology, Beijing, 100124, China \\ ${ }^{2}$ Beijing Key Laboratory of Heat Transfer and Energy, Beijing University of Technology, Beijing, \\ 100124, China
}

\begin{abstract}
In the process of centrifugal dry granulation of blast furnace slag, the thickness of the liquid film at the outlet of the rotor will have a significant impact on the degree of crushing of the slag after leaving the rotor and the size of the solid particles formed. Therefore, this paper uses the VOF method to analyse the flow of molten slag inside the cup. The results have shown that the slag flow has a significant effect on the thickness of the liquid film and the linear relationship is obvious; the thickness of the liquid film decreases with the increase of the diameter of the rotary cup and the rotation speed, and the negative correlation with the diameter of the cup is more obvious; Secondly, as the depth of the cup increases, the thickness of the liquid film decreases first and then increases. The inclination angle of the cup wall has little effect on the thickness of the liquid film.
\end{abstract}

Keywords: Centrifugal granulation of blast furnace slag, Rotary cup, Liquid film thickness, Numerical Simulation.

\section{Introduction}

Since the concept of "carbon neutrality" was put forward, society and research scholars have been paying more attention to how to achieve energy conservation and emission reduction in the steel industry. Therefore, many scholars have proposed to solve the problem of blast furnace slag granulation by centrifugal dry granulation of blast furnace slag by rotary cup or turntable. In 1985, Pickering et al. [1]in the United Kingdom first proposed a rotary cup blast furnace slag centrifugal granulation and waste heat recovery system, which has a compact structure, high heat recovery efficiency and neat slag particles. Subsequently, Mizuochi et al. [2]also conducted cold experiments on three cup types: flat discs, blade discs and wheel discs. The results showed that the atomization effect of the wheel turntable is better, which provides valuable information for the effective recovery of steel slag. Wu et al. [3]studied the effect of different structures of granulators on the granulation effect using water as the experimental working fluid and the results have shown that the rotary cup can produce finer particles.

\footnotetext{
* Corresponding author: jfwang@bjut.edu.cn
} 
Due to the characteristics of excessively high slag temperature and low thermal conductivity, the use of alternatives for hot or cold experiments has certain limitations. Therefore, numerical simulation theoretical analysis has become the goal of many researchers. Pan et al. [4] established a CFD model for the liquid film on the surface of the turntable granulator and pointed out that proper control of the slag temperature, flow rate and granulator speed is beneficial to the granulation effect. Wang et al. [5] established a three-dimensional VOF model based only on free surface flow by using the physical mechanism of slag rotary disc centrifugal atomization, and obtained the process from liquid slag spreading to film formation to granulation. By simulating three rotary cups with different structural parameters, Chen et al. [6]determined that the cup diameter of $200 \mathrm{~mm}$ and depth of $15 \mathrm{~mm}$ are the optimal control parameters that match the specific flow. Chang et al. [7]compared the rough and smooth surface of the turntable through simulation, and found that the smooth surface of the disc is easier to get smaller diameter slag particles, and analyzed the influence of flow rate, viscosity, surface tension, etc. on centrifugal granulation.

At present, since centrifugal technology has not been applied in actual production, most researchers focus on establishing simulation models for simulation theoretical analysis. Based on the research of the above scholars, the flow and diffusion of the liquid film on the rotating cup have not been systematically analyzed. It is necessary to discuss the distribution of the blast furnace slag liquid film during the centrifugal granulation process of the rotary cup. Therefore, this paper uses the VOF method in the CFD software to establish a rotary cup model, and discusses the influence of the slag flow rate and cup structure on the thickness of the liquid film.

\section{Models and methods}

\subsection{Calculation model and meshing}

\subsubsection{Model assumptions.}

In order to simplify the simulation, assume: (1) Ignore the wall thickness of the rotary cup; (2) The molten slag is regarded as an incompressible fluid and the viscosity and density are constant;(3) The slag liquid is constant and continuous during the flow process; (4) The simulated flow phenomenon is regarded as a two-dimensional flow symmetrical on the axis.

\subsubsection{Calculation area and meshing.}

The rotary cup model and meshing used in the simulation are shown in Figure 1 . AB is the symmetry axis of the rotary cup, adopting the rotational symmetry axis as the boundary type to simplify the three-dimensional rotary cup model into a two-dimensional model; BC is the radius of the rotary cup, $\mathrm{CD}$ is the wall of the cup and has a certain inclination angle, both of which use the non-slip cup wall as the boundary condition; DE is the outlet of the rotary cup, using the pressure outlet boundary type; the slag is injected into the rotary cup at a certain mass flow from $\mathrm{AE}$, and the $\mathrm{AE}$ uses the speed inlet as the boundary type. It can be seen from Figure 1 that the grid is divided, and the grid in the area near the interface between the air and the slag phase is encrypted to distinguish the thickness of the slag liquid film. In order to verify the grid independence, the simulation results of four grids of $19,500,27,300,32,500$ and 41,600 were compared by increasing the nodes on the model 
lines and the grids in the vicinity of the dense phase interface. The results of the flow of slag in the rotor under the four numbers of grids are very small. Therefore, considering the accuracy of calculation and the performance of computer equipment and other factors, a number of 32,500 grids is selected for simulation calculation.

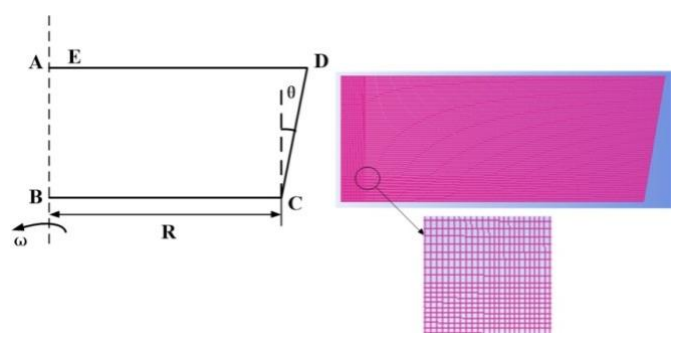

Fig. 1. Rotary cup model and meshing.

\subsection{Mathematical model}

The movement state of the slag in the rotary cup can be described by the conservation of mass equation, the conservation of momentum equation and the conservation of energy equation, which constitute the basic governing equations of fluid mechanics. The VOF method simulates the flow of two or more fluids that do not mix by establishing continuity equations and momentum equations, and realizes the tracking of each calculation cell on the phase interface.

Continuity equation

$$
\nabla \cdot(\rho \vec{v})=0
$$

where $\rho$ is the density, $\mathrm{kg} / \mathrm{m}^{3} ; \vec{v}$ is the velocity vector, $\mathrm{m} / \mathrm{s}$.

Momentum equation

$$
\frac{\partial}{\partial t}(\rho \vec{v})+\nabla \cdot(\rho \overrightarrow{v v})=-\nabla p+\nabla \cdot[\mu(\nabla \vec{v}+\nabla \vec{v})]+\vec{F}
$$

where $p$ is the pressure, $\mathrm{Pa} ; \mu$ is the dynamic viscosity, $\mathrm{Pa} \cdot \mathrm{s} ; \vec{F}$ is the interface force source term, $\mathrm{N} / \mathrm{m}^{3}$.

Each fluid follows the energy conservation equation

$$
\frac{\partial}{\partial t}(\rho H)+\nabla \cdot[\vec{v}(\rho H)+p]=\nabla \cdot(k \nabla T)
$$

where $H$ is the total energy, $\mathrm{J} ; k$ is the thermal conductivity, $\mathrm{W} / \mathrm{mK}$.

\section{Results and discussion}

Figure 2 shows the flow of liquid slag inside the rotary cup. The molten slag enters the rotary and flows to the bottom along the rotational symmetry axis, and then spreads towards the radius of the rotor under the action of centrifugal force, and the thickness of the liquid film first increases and then decreases; the liquid slag starts to accumulate with the circular motion of the rotary cup and close to the walls of the cups on both sides, and flows toward the outlet of the rotor cup. After the balance is reached, the flow conditions on the non-slip wall on both sides of the rotational symmetry axis no longer change, and a relatively stable liquid film thickness is also formed at the outlet of the rotary. 


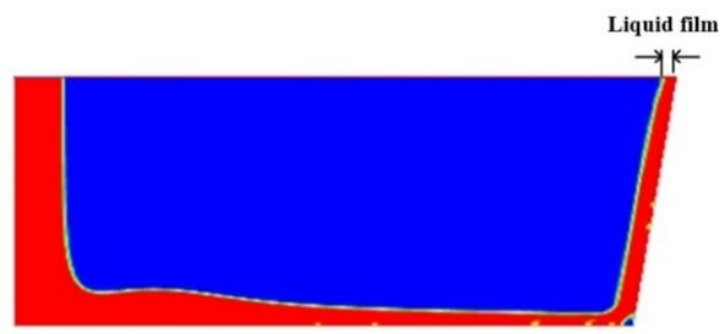

Fig. 2. Flow and spread of molten slag inside the rotary cup.

\subsection{Influence of slag flow rate on liquid film thickness}

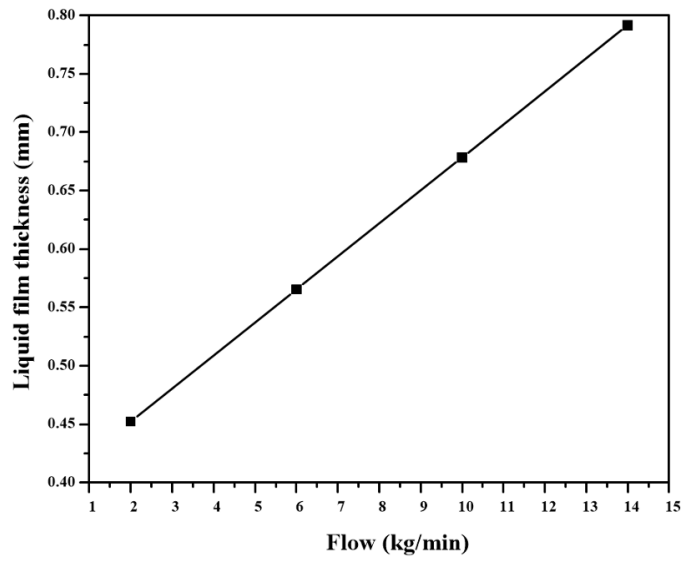

Fig. 3. Influence of different flow rates on liquid film thickness.

Figure 3 shows the variation of liquid film thickness with molten slag injection flow when the rotation speed is $300 \mathrm{r} / \mathrm{s}$, the cup depth is $10 \mathrm{~mm}$, the cup diameter is $50 \mathrm{~mm}$, and the cup wall tilt angle is $10^{\circ}$. It can be seen from Figure 3 that the thickness of the liquid film increases with the increase of the slag flow rate, and the relationship between the two is approximately linear; From the simulation data, when the slag flow rate is $2 \mathrm{~kg} / \mathrm{min}$, the thickness of the liquid film is $0.4521 \mathrm{~mm}$. When the flow rate increases to $14 \mathrm{~kg} / \mathrm{min}$, the thickness increases to $0.7904 \mathrm{~mm}$, an increase of about $75 \%$. This is because a large amount of slag accumulates at the bottom of the rotary cup with a larger flow rate, resulting in excessive slag flowing along the wall of the cup, increased friction, and increased liquid film thickness. Therefore, in order to obtain smaller diameter slag particles, the slag inlet flow should not be too large.

\subsection{Influence of rotation speed on liquid film thickness}

Figure 4 shows the variation of liquid film thickness with rotation speed when the slag flow rate is $6 \mathrm{~kg} / \mathrm{min}$, the depth of the rotor is $10 \mathrm{~mm}$, the diameter of the rotor is $50 \mathrm{~mm}$, and the inclination angle of the cup wall is $10^{\circ}$. It can be seen from Figure 4 that the thickness of the liquid film decreases with the increase of the speed; from the simulation results, when the rotation speed is $200 \mathrm{r} / \mathrm{s}$, the thickness of the liquid film is $0.7915 \mathrm{~mm}$. When the rotation speed is increased to $350 \mathrm{r} / \mathrm{s}$, the thickness decreases to $0.4523 \mathrm{~mm}$, which is $42.86 \%$. This is because the centrifugal force on the rotor cup increases with the increase of the rotation speed, and the linear velocity at the edge of the rotary cup increases, so that the speed of the 
molten slag flowing increases, and the liquid film formed by the molten slag at the outlet of the rotor cup becomes thinner. The smaller liquid film thickness is conducive to the breaking of molten slag and solidification of particles. But after reaching a certain speed, the thickness of the liquid film on the edge of the cup wall at the outlet of the rotor does not change.

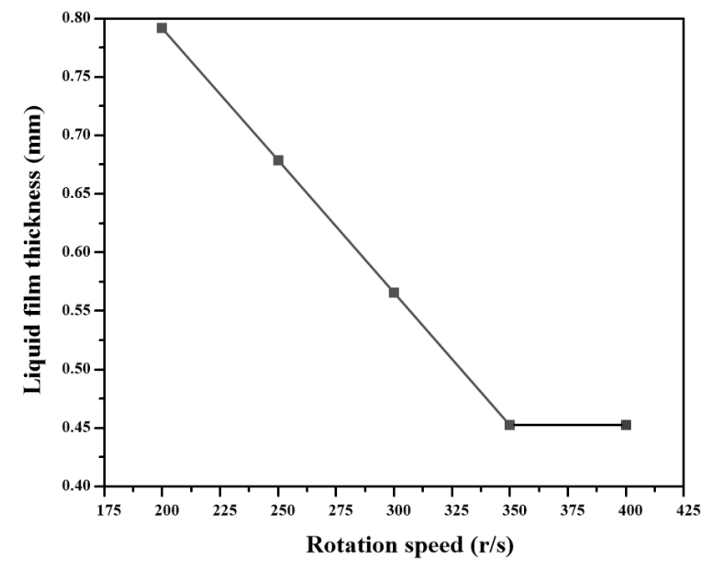

Fig. 4. Influence of different rotation speeds on liquid film thickness.

\subsection{Influence of cup diameter on liquid film thickness}

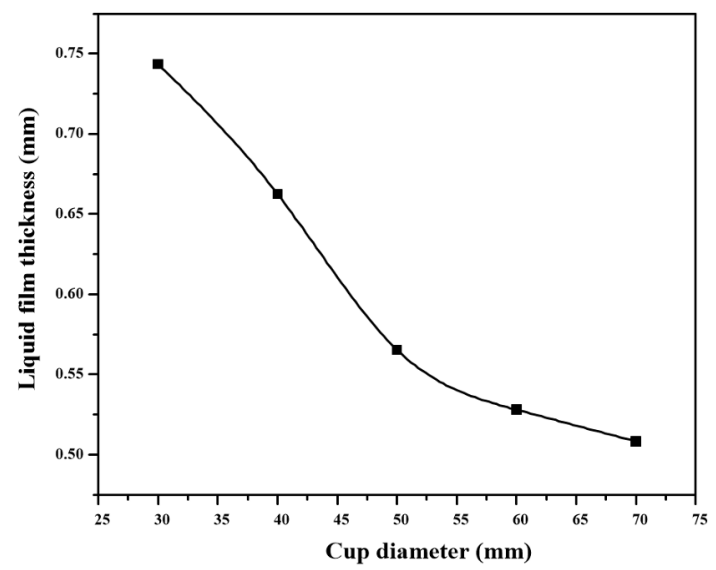

Fig. 5. Influence of different cup diameters on liquid film thickness.

Figure 5 shows the variation of the thickness of the liquid film with the diameter of the cup when the slag flow rate is $6 \mathrm{~kg} / \mathrm{min}$, the rotation speed is $300 \mathrm{r} / \mathrm{s}$, the cup depth is $10 \mathrm{~mm}$, and the cup wall inclination angle is $10^{\circ}$. It can be seen from Figure 5 that as the diameter of the cup increases, the thickness of the liquid film gradually decreases; the simulation results show that when the diameter of the rotary cup increases from $30 \mathrm{~mm}$ to $70 \mathrm{~mm}$, the thickness of the liquid film decreases from $0.7435 \mathrm{~mm}$ to $0.5084 \mathrm{~mm}$, a decrease of approximately $31.62 \%$. This is because as the diameter increases, the linear velocity at the edge of the rotary cup increases, which speeds up the slag speed; and as the diameter increases, the bottom area of the rotary cup increases, the slag wetting area increases, and the flow and spreading become more sufficient, and the thickness of the liquid film on the bottom of the cup and the cup wall becomes smaller. A thinner liquid film is conducive to 
the formation of finer liquid filaments, thereby helping to obtain slag particles with better particle size.

\subsection{Influence of the inclination angle of the rotary cup on liquid film thickness}

Figure 6 shows the variation of liquid film thickness with the diameter of the cup when the slag flow rate is $6 \mathrm{~kg} / \mathrm{min}$, the cup diameter is $50 \mathrm{~mm}$, the rotation speed is $300 \mathrm{r} / \mathrm{s}$, and the cup depth is $10 \mathrm{~mm}$. It can be seen from Figure 6 that as the tilt angle changes, the thickness of the liquid film first decreases and then increases; the simulation results show that the thickness is $0.5851 \mathrm{~mm}$ when the inclination angle is $5^{\circ}$. When the inclination angle increases to $15^{\circ}$, the thickness decreases to $0.5157 \mathrm{~mm}$. When the inclination angle continues to increase, the thickness increases instead, and the thickness increases to $0.5555 \mathrm{~mm}$ when the inclination angle reaches $25^{\circ}$. However, the difference between the maximum liquid film thickness and the minimum liquid film thickness is only $0.0694 \mathrm{~mm}$, so the tilt angle has a limited effect on the thickness.

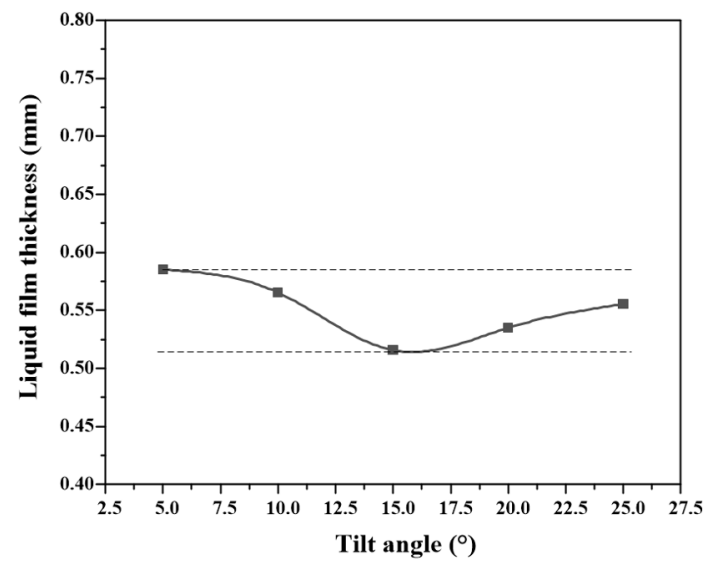

Fig. 6. Influence of different inclination angles on liquid film thickness.

\subsection{Influence of the depth of the cup on the thickness of the liquid film thickness}

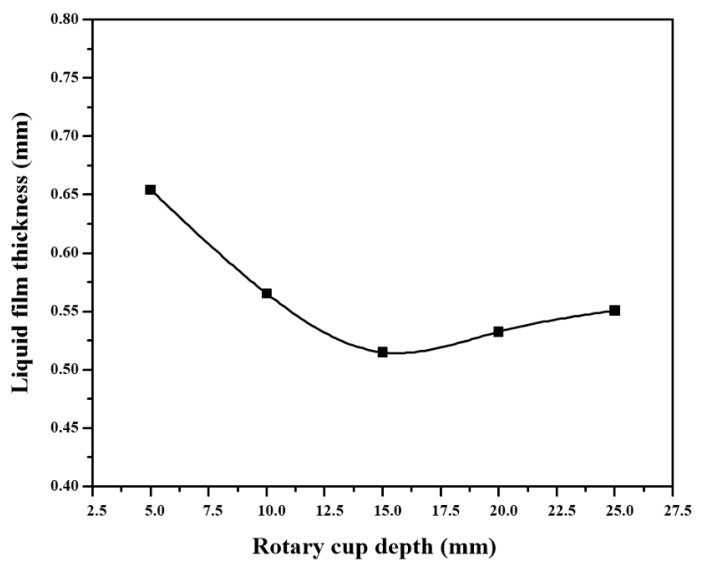

Fig. 7. Influence of different cup depths on liquid film thickness. 
Figure 7 shows the variation of the thickness of the liquid film with the depth of the cup when the slag flow rate is $6 \mathrm{~kg} / \mathrm{min}$, the cup diameter is $50 \mathrm{~mm}$, the rotation speed is $300 \mathrm{r} / \mathrm{s}$, and the cup wall inclination angle is $10^{\circ}$. It can be seen from Figure 7 that as the depth of the cup increases, the thickness of the liquid film first decreases and then increases. The simulation results show that when other parameters remain unchanged, the depth of the cup changes from $5 \mathrm{~mm}$ to $15 \mathrm{~mm}$, and the thickness of the liquid film decreases from $0.6543 \mathrm{~mm}$ to $0.5150 \mathrm{~mm}$. This is because the internal area of the rotary cup is increased due to the increase in depth, and the flow of slag on the wall of the cup before leaving the cup is more sufficient, which is conducive to the formation of slag particles with better particle size. The depth of the cup continues to increase to $25 \mathrm{~mm}$, but the thickness gradually begins to increase. This is because the depth increases to a certain extent that the frictional resistance between the slag and the cup is too large, which causes the flow of the slag to be blocked and the thickness of the liquid film increases instead, which is not conducive to the formation of particles with better particle size.

\section{Conclusion}

Through the numerical simulation of the liquid film formation process of blast furnace slag centrifugal granulation, the influence of each research parameter on the thickness of the liquid film at the outlet of the rotor is obtained.

- The thickness of the liquid film increases with the increase of the slag inlet flow rate (Significant influence and obvious linear relationship), and decreases with the increase of the diameter of the cup and the rotation speed of the cup. With the increase of the cup depth, it first decreases and then increases. The inclination angle of the cup wall has little effect on the thickness of the liquid film.

- The degree of influence of the five research parameters on the thickness of the liquid film in descending order is slag flow, rotation speed, cup diameter, cup depth, and cup inclination angle.

\section{References}

1. Pickering S J, Hay N, Roylance T F, et al. New process for dry granulation and heat recovery from molten blast-furnace slag[J]. Ironmaking and steelmaking, 1985, 12 (1): 14-21.

2. Mizuochi T, Akiyama T. Cold Experiments of Rotary Vaned-disks and Wheels for Slag Atomization[J]. Isij International, 2003, 43(9): 1469-1471.

3. Wu J J, Wang H, Zhu X, et al. Cold experiment of slag centrifugal granulation by rotary atomizer: Effect of atomizer configuration[J]. Applied Thermal Engineering, 2017, 111:1557-1564.

4. Pan Y H, Witt P J, Xie D. CFD simulation of free surface flow and heat transfer of liquid slag on a spinning disc for a novel dry slag granulation process[J]. Progress in Computational Fluid Dynamics An International Journal, 2009, 10(5/6): 292-299.

5. Wang D X, Ling X, Peng H. Simulation of ligament mode breakup of molten slag by spinning disk in the dry granulation process[J]. Applied Thermal Engineering, 2015, 84: 437-447.

6. Chen Y, Ma J, Zhao H, et al. Numerical simulation and experimental study on centrifugal atomization about blast furnace slag[J]. Comput Simul, 2013, 30(2): 235238. 
7. Chang Q, Li X, Ni H, et al. Modeling on dry centrifugal granulation process of molten blast furnace slag[J]. ISIJ International, 2015, 55(7): 1361-1366. 